\title{
RESULTS ON BI-UNIVALENT FUNCTIONS
}

\author{
D. STYER AND D. J. WRIGHT
}

\begin{abstract}
ABSTRACr. When the class $\sigma$ of bi-univalent functions was first defined, it was known that functions of the form $\phi \circ \psi^{-1} \in \sigma$ when $\phi$ and $\psi$ are univalent, map the unit disc B onto a set containing B, and satisfy $\phi(0)=\psi(0)=0, \phi^{\prime}(0)=\psi^{\prime}(0)$. It is shown here that such functions form a proper subset of $\sigma$, and that $\sigma$ is a proper subset of the set of functions of the form $\phi \circ \psi^{-1}$, where $\phi$ and $\psi$ are locally univalent, at most 2-valent, each maps a subregion of $B$ univalently onto $B$, and $\phi(0)=\psi(0)=0, \phi^{\prime}(0)=\psi^{\prime}(0), \psi^{-1}(0)=0$. It is also shown that there are $f(z)=z$ $+a_{2} z^{2}+\cdots$ in $\sigma$ with $\left|a_{2}\right|>4 / 3$. However, doubt is cast that $\left|a_{2}\right|$ can be as large as $3 / 2$.
\end{abstract}

1. Introduction. Let $S$ be the class of normalized univalent functions $f(z)=z+$ $a_{2} z^{2}+\cdots$, and let $C$ denote the complex plane. In 1967, Lewin [2] defined the class $\sigma$ of bi-univalent functions as follows: $h \in \sigma$ if $h \in S$ and $h^{-1} \in S$, i.e., $h^{-1}$ has an analytic extension through the unit disc, $B$, which is univalent on $B$. Through sophisticated use of the Grunsky inequalities, Lewin showed that $\left|a_{2}\right|<$ 1.51 .

Let $\Lambda_{1}$ be the class of functions $\phi(z)=A_{1} z+A_{2} z^{2}+\cdots$ which are univalent in B and satisfy $\phi(B) \supset$ B. Let $\sigma_{1}$ be the collection of functions of the form $\phi \circ \psi^{-1}$ where $\phi, \psi \in \Lambda_{1}$ and $\phi^{\prime}(0)=\psi^{\prime}(0)$. It was pointed out by Nehari (see [2]) that $\sigma_{1} \subset \sigma$, but it was not known if $\sigma_{1}=\sigma$. Suffridge [4] produced a function in $\sigma_{1}$ with $a_{2}=4 / 3$ and conjectured that $\left|a_{2}\right|<4 / 3$ for all functions in $\sigma$. Netanyahu [3] proved that in $\sigma_{1},\left|a_{2}\right|<4 / 3$ is correct.

Let $\Lambda_{2}$ be the class of functions $\phi(z)=A_{1} z+A_{2} z^{2}+\cdots$ such that $\phi$ is locally univalent and at most 2-valent in $B$, and there is a neighborhood, $N$, of zero which $\phi$ maps univalently onto $B$. Let $\sigma_{2}$ be the collection of functions of the form $\phi \circ \psi^{-1}$ on B where $\phi, \psi \in \Lambda_{2}$ and $\phi^{\prime}(0)=\psi^{\prime}(0)$. Here the branch of $\psi^{-1}$ is chosen which sends zero to zero. Clearly $\Lambda_{1} \subset \Lambda_{2}$ and $\sigma_{1} \subset \sigma_{2}$.

In this paper we show that $\sigma_{1} \subsetneq \sigma \varsubsetneqq \sigma_{2}$. Recently Bshouty, Hengartner and Schober [1] have, independently given a function, similar to our Example 1, showing that $\sigma_{1} \neq \sigma$. We also show that there are functions in $\sigma$ with $a_{2}>4 / 3$. Our work leads to several open questions.

2. $\sigma_{1} \subsetneq \sigma \subsetneq \sigma_{2}$. We begin with a function in $\sigma$ which is not in $\sigma_{1}$.

EXAMPLE 1. For $0<\theta<\pi / 2$ consider the functions $h_{\theta} \in S$ given by

$$
h_{\theta}(z)=\cos \theta \frac{z e^{-i \theta}}{1-\left(z e^{-i \theta}\right)^{2}}+\sin \theta\left[\frac{i}{2} \log \frac{1+z e^{-i \theta}}{1-z e^{-i \theta}}\right] \text {. }
$$

Received by the editors February 23, 1979 and, in revised form, June 13, 1980.

AMS (MOS) subject classifications (1970). Primary 30A32, 30A34. 
The image of $\mathbf{B}$ under $h_{\theta}$ is the complex plane less two vertical slits $\left\{w=u_{0}(\theta)+\right.$ $\left.i v: v<v_{0}(\theta)\right\}$ and $\left\{w=-u_{0}(\theta)+i v: v>-v_{0}(\theta)\right\}$, where $u_{0}(\theta)=(\pi / 4) \sin \theta$ and $v_{0}(\theta) \rightarrow+\infty$ as $\theta \rightarrow \pi / 2^{-}$. Let $\theta$ be sufficiently near $\pi / 2$, that $u_{0}(\theta)>\frac{1}{2}$ and $u_{0}(\theta)+i v_{0}(\theta) \notin \mathrm{B}$. Then, by the reflection principle, $h_{\theta}^{-1}$ has an analytic extension from $B \cap\left\{w:|\operatorname{Re} w|<u_{0}(\theta)\right\}$ to $\mathbf{B}$, which is readily seen to be one-one on $\mathbf{B}$. Thus, $h_{\theta} \in \sigma$ if $\theta$ is sufficiently near $\pi / 2$.

Now, suppose $h_{\theta} \in \sigma_{1}$, i.e., $h_{\theta}=\phi \circ \psi^{-1}$ for some $\phi, \psi \in \Lambda_{1}$ with $\phi^{\prime}(0)=\psi^{\prime}(0)$. Since $\phi$ must map $\psi^{-1}(\mathrm{~B})$ onto $h_{\theta}(\mathrm{B})$, and since $h_{\theta}(\mathrm{B})$ is dense in the plane, $\phi$ cannot be one-one on a region larger than $\psi^{-1}(B)$. Thus, $\psi^{-1}(B)=B$ and $h_{\theta}(B)=\phi(B)$. This is impossible since $\mathrm{B} \subset \phi(\mathrm{B})$, but $\mathrm{B} \not h_{\theta}(\mathrm{B})$.

This proof shows, in fact, that if $f \in \sigma_{1}$, then $f(B)$ is not dense in the plane.

In order to show that $\sigma \subset \sigma_{2}$, we shall construct for $h \in \sigma$ a simply-connected, two sheeted Riemann surface, $M$, over $C$ by piecing together $B$ and $h(B)$ along the component of $h(\mathbf{B}) \cap \mathbf{B}$ which contains zero. We then define $\phi: \mathbf{B} \rightarrow \mathbf{C}$ so that $\phi(0)=0$ and the Riemann surface for $\phi^{-1}$ is $M$. It will follow that $\phi \in \Lambda_{2}$ and that $\psi=h^{-1} \circ \phi \in \Lambda_{2}$. The details are given below.

\section{Proposition. $\sigma \subset \sigma_{2}$.}

Proof. Let $h \in \sigma, U_{1}=\{(w, 1): w \in \mathbf{B}\}$ and $U_{2}=\{(w, 2): w \in h(\mathbf{B})\}$. Further, set $(w, 1)=(\tilde{w}, 2)$ if and only if $w=\tilde{w}$ and this point lies in the component, $\Omega$, of B $\cap h(B)$ which contains zero. Let $M=U_{1} \cup U_{2}$ with the topology inherited from C. Define $\pi: M \rightarrow \mathrm{C}$ by $\pi(w, j)=w$ and let $\pi_{j}$ denote the restriction of $\pi$ to $U_{j}$, $j=1,2$. Then $\pi_{i} \circ \pi_{j}^{-1}$ is the identity map where it is defined. Thus, $\left\{\left(\pi_{1}, U_{1}\right),\left(\pi_{2}, U_{2}\right)\right\}$ is an analytic atlas for $M$, and $M$ is a Riemann surface. Also, $M$ is simply connected since $U_{1}$ and $U_{2}$ are, and $U_{1} \cap U_{2}$ is connected. Clearly $M$ is hyperbolic, and thus there is an analytic bijection $g$ from $B$ onto $M$ with $g(0)=(0,1)$. Define $\phi: B \rightarrow C$ by $\phi=\pi \circ g$. Clearly $\phi \in \Lambda_{2}$.

Since $h \in \sigma, h^{-1}$ has an analytic one-one branch on $\mathrm{B}$, which we shall call $h_{1}^{-1}$. Further, we denote by $h_{2}^{-1}$ the one-one analytic branch of $h^{-1}$ on $h(\mathrm{~B})$. Define $\psi: \mathbf{B} \rightarrow \mathbf{C}$ by $\psi(z)=\left(h_{j}^{-1} \circ \pi \circ g\right)(z)$ if $g(z) \in U_{j}, j=1,2$. It is assured that $\psi$ is well defined since $h_{1}^{-1}=h_{2}^{-1}$ on $\Omega$. Clearly $\psi \in \Lambda_{2}$ and $h=\phi \circ \psi^{-1}$. Thus $h \in \sigma_{2}$.

We note that $\phi$ covers $\Omega$ exactly once and $\psi$ covers $h^{-1}(\Omega)$ exactly once. Thus, an extremal function for maximizing $\left|a_{2}\right|$ over $\sigma$ may be found amongst functions in $\Lambda_{2}$ which cover a neighborhood of zero exactly once.

The next example shows that $\sigma$ is a proper subset of $\sigma_{2}$.

EXAMPLE 2. For $\alpha>1$, let $\tau(z)=(z+1)^{\alpha} / 2^{(\alpha-1)}-1, \tau(1)=1$. We show below that there is a neighborhood $N$ of zero such that $\tau \mid N$ is one-one and $\tau(N)=\mathbf{B}$. Let $x_{0}$ be the zero of $\tau$ in $N, g(z)=\left(z+x_{0}\right) /\left(1+x_{0} z\right)$, and consider $\phi=\tau \circ g$. For $2<\alpha<4, \phi \in \Lambda_{2} \backslash \Lambda_{1}$. If $\psi(z)=-\phi(-z)$, then $\psi \in \Lambda_{2}$ and $h=\phi \circ \psi^{-1} \in \sigma_{2}$. However, $h$ fails to be univalent near -1 when $\alpha>2$. In particular, for $\alpha=3, h$ is holomorphic at -1 and has there a critical point of order 2 . Thus, $h \notin \sigma$.

It remains to show that $\tau$ covers B univalently, or equivalently, that $f(z)=$ $(z+1)^{\alpha}$ covers univalently the disc $D$ with center $2^{\alpha-1}$ and radius $2^{\alpha-1}$. Observe that $f\left(e^{i t}\right)=[2 \cos (t / 2)]^{\alpha} e^{i \alpha t / 2}$, and consider $\rho=2^{\alpha} \cos (\alpha t / 2)$, which describes $\partial D$ 
in polar form. It suffices to show that $2^{\alpha} \cos (\alpha t / 2)<[2 \cos (t / 2)]^{\alpha}$ for $0<t<\pi / \alpha$. Letting $\nu(t)=[\cos (t / 2)]^{\alpha}-\cos (\alpha t / 2)$, we have $\nu(0)=0$ and

$$
\nu^{\prime}(t)=\frac{\alpha}{2}\left[\sin \left(\frac{\alpha t}{2}\right)-\cos ^{(\alpha-1)}(t / 2) \sin (t / 2)\right] .
$$

For $0<t<\pi / \alpha, \sin (\alpha t / 2)>\sin (t / 2)$, and consequently $\nu^{\prime}(t)>0$. Thus, $\nu(t)>0$ for $0<t<\pi / \alpha$, and the desired inequality is established.

3. The coefficient problem. If $\phi(z)=A_{1} z+A_{2} z^{2}+\cdots, \psi(z)=B_{1} z+B_{2} z^{2}$ $+\cdots$, and $A_{1}=B_{1}$, then

$$
h(z)=\left(\phi \circ \psi^{-1}\right)(z)=z+\left(\frac{A_{2}-B_{2}}{A_{1}^{2}}\right) z^{2}+\cdots .
$$

If we choose $\psi(z)=-\phi(-z)$, then for $\phi \in \Lambda_{j}, j=1,2$, we have $\psi \in \Lambda_{j}, h \in \sigma_{j}$, and $h(z)=z+\left(2 A_{2} / A_{1}^{2}\right) z^{2}+\cdots$. Thus, to maximize the modulus of the second coefficient of $h$ over $\sigma_{j}$ it is sufficient to solve the extremal problem

$$
\underset{\phi \in \Lambda_{j}}{\operatorname{maximum}}\left|\frac{A_{2}}{A_{1}^{2}}\right| \text {. }
$$

The above choice of $\psi$ relative to $\phi$ automatically yields an $h$ in $\sigma$ when $\phi \in \Lambda_{1}$ $\left(\sigma_{1} \subset \sigma\right)$, but when $\phi \in \Lambda_{2}$ this approach need not produce a univalent $h$. However, when $\psi(z)=-\phi(-z), h^{-1}(z)=-h(-z)$, so if $h \in S$, then $h^{-1} \in S$ and $h \in \sigma$.

Netanyahu [3] showed that the solution of (1) with $j=1$ is $2 / 3$ and that the extremal function maps $B$ onto the plane less the slit $(-\infty,-1]$ and a slit along $|z|=1$ centered at -1 . In Example 3 we generalize this function to obtain a $\phi \in \Lambda_{2}$ with $\left|A_{2} / A_{1}^{2}\right|>2 / 3$. The function $h$ so generated will be univalent for certain values of the parameters and hence, as discussed above, will be in $\sigma$.

Example 3. Suppose $0<\theta<\pi, 2-(\theta / \pi)<\alpha<4$, and let $\mathbf{B}^{+}=\{z \in \mathrm{B}: \operatorname{Im} z$ $>0\}$. Consider $\lambda(z)=z^{\alpha}, \lambda(1)=1$, followed by $S(z)=e^{-i \theta}\left(z-e^{i \theta}\right) /\left(z-e^{-i \theta}\right)$. The first function sends $B^{+}$into $B$, and the second one maps $B$ onto the upper half-plane, sending radial segments $\left\{r e^{i t}: 0<r<1\right\}$ onto circular arcs from $e^{i \theta}$ to the real axis, meeting the real axis orthogonally. The arc $\left\{e^{i t}: 0<t<\right.$ $(2 \pi-\theta) / \alpha\}$ is mapped by $S \circ \lambda$ onto $(-1, \infty)$, with $e^{i \theta / \alpha}$ going to 0 . If $g$ is a self-mapping of $\mathbf{B}^{+}$which sends -1 to 1,0 to $e^{i \theta / \alpha}$, and 1 to $e^{i(2 \pi-\theta) / \alpha}$, then $S \circ \lambda \circ g$ maps $(-1,1)$ to $(-1, \infty)$ and 0 to 0 . Extending to $\mathbf{B}$ via the reflection principle gives the desired function $\phi$. We note that $\phi \in \Lambda_{1}$ if $0<\alpha<2$ and $\phi \in \Lambda_{2}$ for $\alpha>2$.

To obtain the function $g$, let $\mu(z)=[(1+z) /(1-z)]^{2}$ and let

$$
T(z)=(a z+b-a) / z,
$$

where $a=\mu\left(e^{i(2 \pi-\theta) / \alpha}\right)$ and $b=\mu\left(e^{i \theta / \alpha}\right)$. Then $g=\mu^{-1} \circ T \circ \mu$.

Let $\zeta=\phi^{-1}\left(e^{i \theta}\right)=\mu^{-1}((b-a) /(1-a))$. We shall consider only the case $\zeta=i$, i.e., $(b-a) /(1-a)=\mu(i)=-1$. This expresses $\theta$ implicitly as a continuous function of $\alpha$ in an interval about $\alpha=2$ via the equation

$$
m(\theta, \alpha)=\cot ^{2}\left(\frac{\theta}{2 \alpha}\right)-2 \cot ^{2}\left(\frac{2 \pi-\theta}{2 \alpha}\right)=1 .
$$


Thus, we obtain one parameter families of functions $g_{\alpha}, T_{\alpha}, \phi_{\alpha}$. If $\alpha=2$, then $\theta=2 \arccos (1 / 3)$ and $\phi_{2}$ is the extremal function for (1) with $j=1$. Assume hereafter that $\alpha$ is larger than 2 but near 2 .
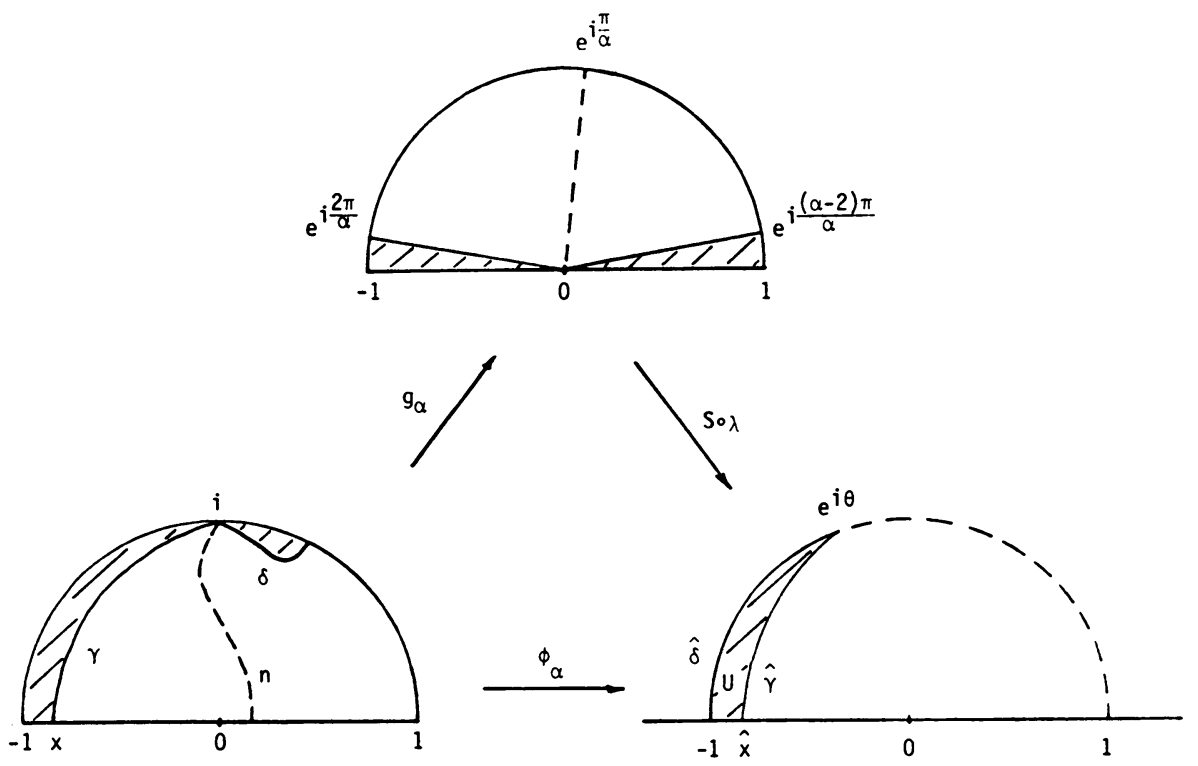

FiguRE 1

Let $\hat{\delta}$ and $\hat{\gamma}$ denote the images of $[0,1]$ and $[-1,0]$, respectively, under $S \circ \lambda$. Then $\hat{\delta}=\left\{e^{i t}: \theta<t<\pi\right\}$, and $\hat{\gamma}$ is a circular arc joining $e^{i \theta}$ and a point, $\hat{x}$, on the real axis. The curves $\hat{\delta}, \hat{\gamma}$, and $[-1, \hat{x}]$ bound the region, $U$, in the upper half-plane which is covered twice by $\phi_{\alpha} \mid \mathbf{B}^{+}$. An examination of the mapping $\phi_{\alpha}$ shows that $\phi_{\alpha}^{-1}(U)$ consists of two components as illustrated by the shaded regions in Figure 1. The curves $\gamma, \delta, \eta$, in Figure 1 correspond to $\hat{\gamma}, \hat{\delta}$ and $\left\{e^{i t}: 0<t<\theta\right\}$, respectively, and $\phi_{\alpha}(x)=\hat{x}$. If $V$ is the region bounded by $\gamma,[x, 1]$, and $\left\{e^{i t}: 0<t<\pi / 2\right\}$, then $\phi_{\alpha} \mid V$ is one-one and $\phi_{\alpha}(V)$ is the upper half-plane less $\hat{\gamma}$. The restriction of $\phi_{\alpha}$ to the region, $W$, bounded by $\left\{e^{i t}: \pi / 2<t<\pi\right\},[-1, y]$ and $\eta$ is univalent, and $\phi_{\alpha}(W)=\mathbf{B}^{+}$. Let $\psi_{\alpha}(z)=-\phi_{\alpha}(-z), h_{\alpha}=\phi_{\alpha} \circ \psi_{\alpha}^{-1}$ and $\tilde{\eta}$ be the reflection of $\eta$ in the imaginary axis. Now, $\psi_{\alpha}$ is one-one on $-\bar{W}=\{-\bar{z}: z \in W\}$, and $\psi_{\alpha}(-\bar{W})=\mathbf{B}^{+}$. Thus, to see that $h_{\alpha}$ is univalent, it is sufficient to show that $-\bar{W} \subset V$. We will do this by showing that for $\alpha$ sufficiently near 2 the curves $\gamma$ and $\tilde{\eta}$ intersect only at $i$.

Since $a$ and $b$ are continuous functions of $\alpha$, one can show that as $\alpha$ approaches 2, $T_{\alpha}^{-1}$ approaches $T_{2}^{-1}$ uniformly relative to the chordal metric on the closed upper half-plane. It then follows that $g_{\alpha}^{-1}$ approaches $g_{2}^{-1}$ uniformly on the closure of $\mathbf{B}^{+}$.

Let $\eta_{2}=g_{2}^{-1}([0, i])$ and $\gamma_{2}=g_{2}^{-1}([0,1])$. By reflection $g_{2}^{-1}$ is conformal at zero, so $\eta_{2}$ and $\gamma_{2}$ meet at $i$ with angle $\pi / 2$. There is a neighorhood $\Re$ of zero and narrow sectors with vertices at zero, symmetric about $[0, i]$ and $[0,1]$, respectively, such that the images of these sectors under $g_{2}^{-1} \mid \Re$ have positive angular separation at $i$. Since $g_{\alpha}^{-1}$ approaches $g_{2}^{-1}$ uniformly on $\Re$ as $\alpha$ approaches 2 , there are choices of $\arg g_{\alpha}^{-1}$ and $\arg g_{2}^{-1}$ such that $\arg g_{\alpha}^{-1}$ approaches $\arg g_{2}^{-1}$ uniformly as $\alpha$ approaches 
2. It follows that for $\alpha$ near 2 the images of the sectors under $g_{\alpha}^{-1}$ have positive angular separation at $i$. Thus, in some neighborhood of $i, \gamma$ and $\tilde{\eta}$ cannot intersect except at $i$ for all $\alpha$ sufficiently near 2 . Outside this neighborhood, $\gamma_{2}$ and $\tilde{\eta}_{2}$ are a positive distance apart, and consequently so are $\gamma$ and $\tilde{\eta}$ when $\alpha$ is sufficiently near 2.

Extensive but routine calculations show that for these functions $\phi_{\alpha}$,

$$
\begin{aligned}
\frac{A_{2}}{A_{1}^{2}}= & \frac{\sin \theta}{\alpha \sin (\theta / \alpha)}\left\{1+\cos (\theta / \alpha)-\frac{1}{2}\left[\frac{\sin (\pi / \alpha)}{\sin ((\pi-\theta) / \alpha)}+\frac{\sin ((\pi-\theta) / \alpha)}{\sin (\pi / \alpha)}\right]\right\} \\
& -\cos \theta .
\end{aligned}
$$

At the point $\alpha=2, \theta=\theta_{0}=2 \arccos (1 / 3)$, the level set $m(\theta, \alpha)=1$ given by (2) has slope $d \alpha / d \theta=3 /[3 \arccos (1 / 3)-\pi] \sim 5.44$. Thus

$$
\vec{v}=(1,3 /[3 \arccos (1 / 3)-\pi])
$$

is a vector tangent to the level curve (2) at $\left(\theta_{0}, 2\right)$ in the direction of increasing $\alpha$. Further computation shows that the gradient of $A_{2} / A_{1}^{2}$ at $\left(\theta_{0}, 2\right)$ is $(.0112 \ldots, 0)$, and consequently the directional derivative of $A_{2} / A_{1}^{2}$ in the direction $\vec{v}$ is $.002 \ldots$ Thus, for $\alpha$ slightly larger than $2, A_{2} / A_{1}^{2}$ takes values strictly larger than $2 / 3$. Using a programmable calculator with $\alpha=2.1$ we obtain $\theta=2.4785 \ldots$ and $A_{2} / A_{1}^{2}=.6674 \ldots$

It is an open question as to whether the maximum of $\left|a_{2}\right|$ over $\sigma$ is as large as that over $\sigma_{2}$. In regard to the latter problem, we show by Example 4 below that the solution of (1) with $j=2$ is at least 0.7236 .

EXAmple 4. Consider

$$
f(z)=-z \frac{(1+b) z-2 b}{(1+b)-2 b z}\left[\frac{1-b z}{z-b}\right]^{2}, \quad 0<b<1 .
$$

We note that $f(\partial \mathbf{B}) \subset \partial \mathbf{B}$, since $f$ is a product of functions with this property. Also, $f(\bar{z})=\overline{f(z)}$. Calculations show

$$
f^{\prime}(z)=\frac{-2 b^{2}(1+b)(1-b z)(1-z)^{2}\left[z^{2}+(1-3 b) z+1\right]}{(1+b-2 b z)^{2}(z-b)^{3}} .
$$

We see that $f$ has a pole of order 2 at $b$, a zero of order 2 at $1 / b$, a critical point of order 2 at 1 and simple critical points at the roots, $z_{0}$ and $\bar{z}_{0}$, of $z^{2}+(1-3 b) z+1$ $=0$. These account for all the branch points of the image of $f$. Assume $\operatorname{Im} z_{0}>0$ and note that $\left|z_{0}\right|=1$. Taking these features into consideration we conclude that $f$ maps $B$ univalently onto the plane less the slit $[-1, \infty)$ and the slit along $|z|=1$ from -1 to $f\left(z_{0}\right)$. Furthermore, $f$ maps $[-1, b)$ onto $[-1, \infty)$. Let $g$ be a self-mapping of $\mathrm{B}^{+}$which sends -1 to $-1,0$ to 0 , and 1 to $b$, and let $\phi=f \circ g$. Then $\phi\left(\mathbf{B}^{+}\right)=f\left(\mathbf{B}^{+}\right)$, and $\phi$ sends $(-1,1)$ onto $(-1, \infty)$. Extending by the reflection principle to $B$ we see that $\phi \in \Lambda_{2}$. The function $g$ is given by $\mu^{-1} \circ T \circ \mu$, where $\mu(z)=[(1+z) /(1-z)]^{2}$ and $T(z)=\mu(b) z /[z+\mu(b)-1]$.

After considerable calculation we obtain $A_{2} / A_{1}^{2}=\left(-5 b^{3}+3 b+2\right) / 4$, which has a maximum value over $(0,1)$ of $(1+\sqrt{5}) / 2 \sqrt{5}=0.7236 \ldots$, occurring at $b=1 / \sqrt{5}$. 
This leads to the following natural questions. Is $\left|A_{2} / A_{1}^{2}\right|<(1+\sqrt{5}) / 2 \sqrt{5}$ for all $\phi \in \Lambda_{2}$ ? If so, then $\left|a_{2}\right|<(1+\sqrt{5}) / \sqrt{5} \sim 1.45$ for all $f \in \sigma_{2}$, and $\left|a_{2}\right|$ would likely be even less for $f \in \sigma$. In any case, the gap between $4 / 3^{+}$and $1.51^{-}$for $\left|a_{2}\right|$ when $f \in \sigma$ seems rather large.

\section{REFERENCES}

1. D. Bshouty, W. Hengartner and G. Schober, Estimates for the Koebe constant and the second coefficient for some classes of univalent functions (preprint).

2. M. Lewin, On a coefficient problem for bi-univalent functions, Proc. Amer. Math. Soc. 18 (1967), 63-68.

3. E. Netanyahu, The minimal distance of the image boundary from the origin and the second coefficient of a univalent function in $|z|<1$, Arch. Rational Mech. Anal. 32 (1969), 100-112.

4. T. J. Suffridge, A coefficient problem for a class of univalent functions, Michigan Math. J. 16 (1969), $33-42$.

Department of Mathematical Sciences, University of Cincinnati, Cincinnati, Ohio 45221 